\title{
Análise biomecânica ex vivo de dois métodos de osteossíntese de fratura diafisária transversal em fêmur de cães
}

\author{
Biomechanical analisis ex vivo of two osteosynthesis methods for transversal \\ diaphiseal fracture in canine femur
}

\begin{abstract}
Ademar Luiz Dallabrida ${ }^{1}$ João Eduardo Schossler ${ }^{2}$ Eduardo Santiago Ventura de Aguiar ${ }^{1}$ Gustavo Frassetto Amendola ${ }^{1}$ José Henrique Souza da Silva ${ }^{3}$ José Mario Doleys Soares ${ }^{4}$
\end{abstract}

\section{RESUMO}

O presente trabalho teve como objetivo comparar ex vivo, em 12 pares de ossos, a ação das forças de compressão axial e de flexão em dois métodos de fixação de fratura diafisária transversal em fêmur de cães com peso entre 5 e $10 \mathrm{~kg}$. Um com a utilização de dois pinos intramedulares de Steinmann de 2,0mm de diâmetro e outro com a utilização de um método de transfixação cortical interna com pinos de Steinmann de 2,0mm de diâmetro, de forma que os mesmos formem uma tala ao redor do osso e fixando-os com fios de aço inoxidável de 0,6mm de diâmetro em cerclagem. Os ossos fraturados e estabilizados após redução dos fragmentos, foram submetidos às forças em uma prensa Soloteste ${ }^{\circledR}$, sendo que os do grupo G2 (transfixação esquelética interna) apresentaram superioridade estatisticamente significativa, quanto à rigidez do dispositivo de fixação, em relação ao grupo $G 1$ (pino intramedular).

Palavras-chave: cirurgia, ortopedia, cerclagem, força, pino.

\section{ABSTRACT}

The aim of this study was to compare ex vivo, in 12 pars of bone, the axial compression and bending strength of two transversal diaphiseal fracture reducing devices in canine femur of dogs weighting 5 to $10 \mathrm{~kg}$. One method using two intramedullary pins of Steinmann $2.0 \mathrm{~mm}$ diameter, and the other using cortical internal transfixator with two intramedullary pins of Steinmann 2.0mm diameter, which posteriorly turnedup around the bone like a splint and fixed with surgeon wire cerclage $0.6 \mathrm{~mm}$ diameter, to fix them. The fractured and stabilized bones, after the fragments reduction, were put on test under the strength of a Soloteste ${ }^{\circledR}$ press, where the ones from group G2 (internal skelethic transfixation) presented a statistically significant superiority of the fixation device stiffness in relation to those from group $G 1$ (intramedullary pin).

Key words: surgery, orthopedics, cerclage, strength, pin.

\section{INTRODUÇÃO}

Vários dispositivos de fixação interna de fraturas femorais estão descritos na literatura, como colocação de fios de aço ortopédico em cerclagem, placa e parafuso, pinos intramedulares e fixação esquelética externa, pinos travados e combinações destas. Fraturas diafisárias transversais são por vezes reparadas com um pino intramedular, embora se relate sucesso com este método, também se descrevem falhas, principalmente devido à falta de estabilidade rotacional (GILMORE, 1996). A vantagem biomecânica

${ }^{1}$ Médico Veterinário, Discente do Programa de Pós-graduação em Medicina Veterinária (PPGMV), Universidade Federal de Santa Maria (UFSM). Rua Agostinho Malinverne 50, apto 51, 88502-260, Lages, Santa Catarina. E-mail: adallabrida@yahoo.com.br. Autor para correspondência.

${ }^{2}$ Médico Veterinário, Doutor, Docente do Departamento de Clínica de Pequenos Animais, UFSM.

${ }^{3}$ Engenheiro Agrônomo, PhD, Docente do Departamento de Zootecnia, UFSM.

${ }^{4}$ Engenheiro, Doutor, Docente do Departamento de Transportes, UFSM. 
de pinos intramedulares é sua resistência a cargas de envergamento (flexão) porém são poucos resistentes a cargas axiais ou de rotação (FOSSUM, 2002).

As quatro forças fisiológicas primárias que atuam sobre o osso são compressão axial, tensão axial, envergamento (flexão) e torção. Cada uma destas forças isoladamente ou em conjunto, resulta num padrão complexo de pressões e deformações internas no âmbito do osso (HULSE \& HYMAN, 1998).

Fios de aço inoxidável usados em cerclagem, são utilizados para fazer a redução de fragmentos da fratura. Para se efetuar isso com sucesso, eles devem resistir às cargas a que são submetidos nos ossos fraturados. Suas habilidades em resistir a estas cargas são relatadas como tensão gerada durante a sua torção, compressão das superfícies dos fragmentos e a segurança do nó (ROE , 1997).

Quando a fratura ocorre no fêmur ou na tíbia, o implante deve absorver o estresse induzido pela ação da carga axial, para permitir a reabilitação do paciente. Existem vários tipos de implantes para a redução cirúrgica de fraturas de ossos longos e a sua seleção não é tão óbvia. Por esta razão, ortopedistas devem consultar estudos comparativos do comportamento biomecânico dos ossos longos intactos e com implantes (DAMIÁN et al., 2003).

Pesquisas sobre as propriedades dos ossos longos dos cães devem ser feitas para melhor entender as forças e os movimentos que o esqueleto apendicular deve resistir. Este tipo de estudo pode permitir aos cirurgiões, substanciais avanços nas fixações de fraturas e aumentar o seu entendimento do remodelamento ósseo e da ocorrência de fratura com relação ao exercício e o trauma (MARKEL et al., 1994).

Testes biomecânicos têm se tornado cada vez mais comuns para se avaliar a performance dos dispositivos de fixação. Cargas fisiológicas são aplicadas aos implantes para avaliar sua estabilidade mecânica (SCHMIDT et al., 2003).

Exemplo disto é o estudo de BERNARDE et al. (2001), que objetiva comparar a resistência entre dois métodos de fixação óssea (pinos intramedulares e placas de compressão dinâmica). Para tal experimento, foram utilizados 20 fêmures caninos, separados em dois grupos. As forças aplicadas foram de arqueamento crânio-caudal e carga axial excêntrica. Como resultado, foi verificado que ambos se comportaram de forma semelhante no que diz respeito ao esforço de arqueamento, enquanto que a fixação por meio de pinos intramedulares foi superior em compressão e esforço transversal. Como conclusão, foi observada uma rigidez estrutural inferior no modelo utilizando placas metálicas.
Este trabalho tem por objetivo avaliar por comparação a técnica de dois pinos intramedulares, um método alternativo de osteossíntese femural diafisária em cães através da análise biomecânica dos efeitos das forças de compressão axial e de flexão aplicados mediante uma prensa Soloteste ${ }^{\circledR}$, utilizada rotineiramente para o teste de resistência de materiais.

\section{MATERIAL E MÉTODOS}

Foram utilizados 12 pares de fêmures caninos adultos, de cadáveres com, no máximo, 24 horas do óbito, com peso entre 5 e $10 \mathrm{~kg}$, coletados no setor de Patologia Veterinária da Universidade Federal de Santa Maria (UFSM). As peças foram acondicionadas em bolsas plásticas individuais e congeladas em freezer doméstico à temperatura de $\left(-20^{\circ} \mathrm{C}\right)$. O descongelamento foi realizado em geladeira à temperatura de $\left(8^{\circ} \mathrm{C}\right)$ por 12 horas antes de sua utilização.

Os ossos foram distribuídos em dois grupos sendo que os fêmures direitos constituíram o grupo G1 (Pino Intramedular) e os fêmures esquerdos o grupo G2 (Transfixação Esquelética Interna). Cada grupo foi dividido em mais dois subgrupos, compressão axial e flexão, contendo seis pares de ossos cada um.

Cada par de ossos foi transversalmente seccionado, com uma serra manual de cortar canos, na região diafisária, ao mesmo tempo, de forma que se obtivessem quatro segmentos de tamanhos iguais, para que fossem minimizadas as diferenças em decorrência do corte. No grupo G1, foram colocados dois pinos intramedulares de Steinmann de 2,0mm de diâmetro de forma retrógrada, conforme técnica já descrita (PIERMATTEI \& FLO, 1999).

No grupo G2, foram utilizados dois pinos de Steinmann de 2,0mm de diâmetro, fazendo com o primeiro uma transfixação cortical, dupla (proximal e distal) de forma que se obtivesse uma orientação crânio-caudal (Figura 1a). A etapa seguinte consistiu no dobramento da extremidade proximal do pino em sentido distal e elaboração de dois orifícios (proximal e distal) com orientação latero-medial do osso. Outro pino foi dobrado em formato de "U" para colocação nestes orifícios (Figura 1b). Em seguida, as extremidades do pino foram colocadas nos orifícios (Figura 1c). Durante a montagem do dispositivo de transfixação esquelética interna, utilizaram-se abraçadeiras de náilon ${ }^{c}$ esterilizadas em autoclave (Figuras 1a, 1b e 1c), para se fazer a imobilização e o alinhamento temporários da fratura, até que fosse completada a montagem do dispositivo. Após a colocação de todos os pinos, foi efetuada a imobilização definitiva dos mesmos aos segmentos femorais com fios de aço inoxidável de $0,6 \mathrm{~mm}$ de diâ- 


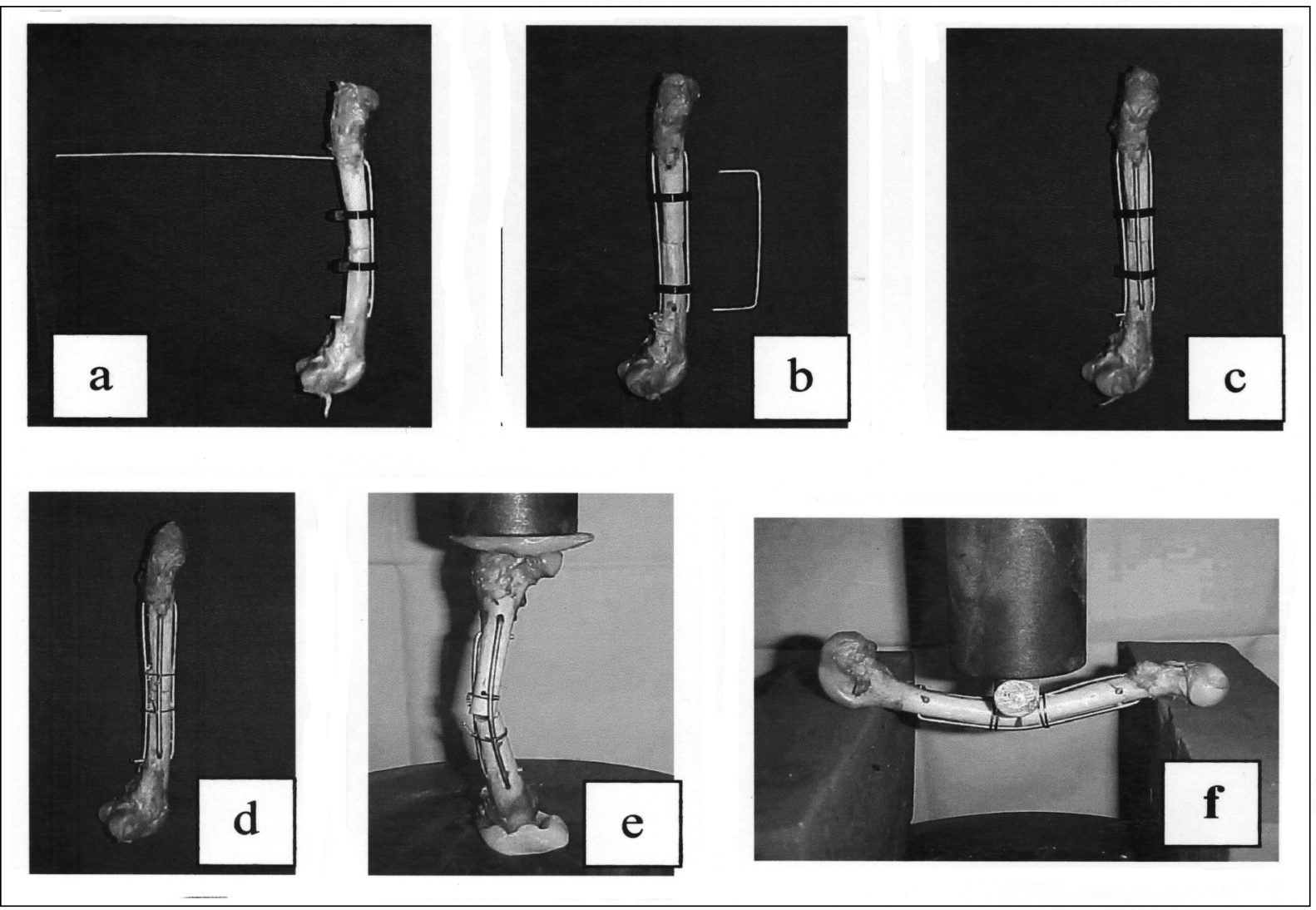

Figura 1 - Método gráfico para a osteossíntese de fratura transversal em fêmur de cão: colocação do pino com orientação crâniocaudal, com a sua extremidade mais longa preparada para ser dobrada distalmente. Notar imobilização temporária com abraçadeiras de náilon; (b) orifícios confeccionados com orientação latero-medial e pino confeccionado em formato de "U", para posterior colocação nestes orifícios; (c) novas abraçadeiras abrangendo o osso e as porções anterior, posterior e lateral dos pinos; (d) final da elaboração do dispositivo de transfixação esquelética interna. Notar a colocação das cerclagens abrangendo o osso e as porções anterior, posterior e lateral dos pinos; (e) osso com dispositivo de transfixação esquelética interna, recebendo a força de compressão axial na prensa Soloteste ${ }^{\circledast}$. Notar o curvamento do osso no sentido cranio-lateral; (f) osso com dispositivo de transfixação esquelética interna, recebendo a força de flexão na prensa Soloteste ${ }^{\circledast}$. Notar o envergamento do osso no sentido caudo-cranial.

metro em cerclagem colocadas cada uma à distância de $1,0 \mathrm{~cm}$ da linha de fratura e retiradas as abraçadeiras (Figura 1d).

Em seguida, os ossos foram levados para o setor de Engenharia da UFSM e as forças de compressão axial (Figura 1e) e flexão (Figura 1f) aplicadas mediante uma prensa Soloteste ${ }^{\circledR}$, com os resultados da resistência de cada implante de estabilização anotados em uma planilha para posterior conversão dos valores em kgf, através da fórmula fornecida pelo setor de Engenharia, conforme, $\mathrm{P} \mathrm{em} \mathrm{kgf}=1,3723 \times \mathrm{L}+5,35$, onde: $\mathrm{P}=$ peso, $\mathrm{kgf}=$ quilograma força, $\mathrm{L}=$ leitura obtida no marcador da prensa.

Durante a elaboração dos testes, a marcação dos valores obtidos na prensa deu-se através da observação de um marcador acoplado à mesma. A par- tir do momento que se exercia pressão nos ossos com os dispositivos de fixação e não havia um aumento da resistência por parte dos mesmos, se considerava que o dispositivo havia chegado na sua força máxima e seus valores anotados.

Para se ter uma adequada acomodação dos fragmentos de cada osso na prensa e evitar o seu deslizamento durante o teste de compressão axial, foram elaboradas duas bases em Jet ${ }^{\mathrm{b}}$ acrílico auto polimerizável (Figura 1e), uma para a porção proximal e outra para a porção distal. Para o teste de flexão, os ossos foram colocados em posição horizontal sobre dois anteparos e a aplicação da força foi realizada no sentido caudo-cranial (Figura 1f). O tempo necessário para a confecção dos dispositivos de fixação foi anotado para posterior comparação entre eles.

Ciência Rural, v.35, n.1, jan-fev, 2005. 
Os resultados obtidos foram estatisticamente analisados por Análise de Variância (ANOVA) e teste F. Os dados foram interpretados, após a transformação em raiz quadrada, em pacote estatístico SAS, e o nível crítico de significância foi de $5 \%(\mathrm{P}>0,05)$.

\section{RESULTADOS E DISCUSSÃO}

A cirurgia experimental veterinária é de extrema importância para a descoberta de novos procedimentos que possam ser utilizados tanto em animais como em humanos. Essa área da medicina veterinária deve interagir com outras áreas afins visando revelar à comunidade científica resultados imparciais e comprovadamente realizados.

É fato conhecido que um material implantado cirurgicamente em um ser vivo será submetido a diversos esforços, tais como tração, rotação, estiramento, deslizamento, compressão, entre outros. Sendo assim, é importante que o cirurgião saiba que sua técnica preconizada apresentará além de boa aceitação, também condições estruturais adequadas. Por isso, a importância do uso de testes biomecânicos em materiais e técnicas que serão respectivamente implantados e aplicados em animais.

Os resultados obtidos estão relacionados na tabela 1 e tabela 2 e demonstraram superioridade altamente significativa quanto à rigidez do dispositivo de transfixação esquelética interna em relação ao pino intramedular e conforme citou FOSSUM (2002), isto ocorreu em função dos pinos intramedulares apresentarem desvantagens biomecânicas quanto à resistência a cargas axiais (compressivas) ou rotacionais.

Os ossos apresentaram comprimento com média de $13,54 \mathrm{~cm}(11,4 \pm 16,5 \mathrm{~cm})$. O tempo necessário para a elaboração dos dispositivos pino intramedular e

Tabela 1 - Resultado do teste biomecânico dos fêmures de cães submetidos a osteotomia diafisária transversal e fixados através dos métodos de pino intramedular (PIM) e transfixação esquelética interna (TfxInt), submetidos às forças de compressão axial e flexão (em kgf) em uma prensa Soloteste ${ }^{\circledR}$.

\begin{tabular}{lccccc}
\hline \multicolumn{3}{c}{ Compressão axial } & \multicolumn{3}{c}{ Flexão } \\
\hline Osso & PIM & TfxInt & Osso & PIM & TfxInt \\
01 & 36,912 & 101,722 & 01 & 30,051 & 80,826 \\
02 & 49,263 & 94,549 & 02 & 16,328 & 54,752 \\
03 & 64,358 & 163,164 & 03 & 10,839 & 30,051 \\
04 & 34,168 & 115,130 & 04 & 17,700 & 34,168 \\
05 & 17,700 & 50,635 & 05 & 16,328 & 31,423 \\
06 & 19,073 & 94,549 & 06 & 17,700 & 45,146 \\
\hline
\end{tabular}

transfixação esquelética interna foram respectivamente de $5,36 \mathrm{~min}(4,3 \pm 6,5 \mathrm{~min})$ e $21,4 \mathrm{~min}(16,3 \pm 26,3 \mathrm{~min})$.

Nos ossos menores, houve uma maior dificuldade em se elaborar o dispositivo de transfixação esquelética interna com o pino de $2,0 \mathrm{~mm}$ de diâmetro, em decorrência da dificuldade de dobrá-lo e colocar as suas extremidades dentro dos orifícios preparados previamente; em contrapartida, nestes ossos, a resistência às cargas mostrou-se muito maior (Tabela 1), sendo que, no grupo G2, um osso desprendeu-se da prensa sem que o dispositivo sofresse qualquer alteração em sua conformação.

Após a montagem dos dispositivos intramedulares no grupo G1, nenhum dos ossos apresentou alinhamento perfeito da linha de fratura, porque o fêmur apresentou uma curvatura anatômica normal; e os pinos, quando inseridos no canal medular, não possibilitaram a perfeita coaptação das bordas da fratura. No entanto, no grupo G2 os ossos se mantiveram alinhados e com compressão na linha de fratura.

Durante o envergamento dos ossos do grupo G2 (compressão axial), houve o desprendimento da ponta do pino de seu orifício na porção cranio-distal do osso, porque neste ponto o pino foi deixado curto, apenas transpassado duas corticais.

Durante o teste de compressão axial observou-se que os ossos do grupo G2 apresentaram a deformidade no sentido crânio-lateral (Figura 1e), apresentando um aspecto convexo, demonstrando que, no fêmur, esta orientação é a que mais gera tensão sobre o osso, conforme citaram HULSE \& HYMAN (1998). Já no grupo G1, o curvamento dos ossos não apresentou nenhuma orientação definida, todos dobrando em direções diferentes. Isso porque os pinos intramedulares

Tabela 2 - Análise estatística dos fêmures de cães submetidos ao teste biomecânico para os métodos de fixação de fraturas de pino intramedular (PIM) e transfixação esquelética interna (TfxInt), através do teste das forças de compressão axial e flexão em uma prensa Soloteste ${ }^{\circledR}$

\begin{tabular}{ccc}
\hline $\begin{array}{c}\text { Dispositivo de } \\
\text { fixação de fratura }\end{array}$ & Compressão axial & Flexão \\
\hline PIM & $36,912^{*}$ & $18,157^{*}$ \\
TfxInt & $103,291^{*}$ & $46,061^{*}$ \\
Média & $70,101^{*}$ & $32,109^{* *}$ \\
F & 18,23 & 15,59 \\
P $<$ & 0,0016 & 0,0027 \\
CV $(\%)$ & 20,872 & 19,84 \\
\hline
\end{tabular}

F - teste f; CV - coeficiente de variância; * - média aritmética dos tratamentos (Tabela 1); ** - média universal (entre os tratamentos).

Ciência Rural, v.35, n.1, jan-fev, 2005. 
não apresentaram uma estabilização adequada quando sob ação das forças axiais.

Testes biomecânicos de forças são largamente utilizados e aceitos para avaliar efetivamente os dispositivos de fixação ortopédica (SCHMIDT et al., 2003). Fatores que afetam os resultados dos testes biomecânicos em ossos devem ser considerados durante o procedimento. Os efeitos da variação entre os ossos quanto à idade, porosidade, tamanho, mineralização e geometria devem ser minimizados (SUKHIANI, 1997). Neste trabalho, procurou-se diminuir essas diferenças utilizando-se cães adultos e ambos os fêmures do mesmo animal, sendo desta forma um deles o controle do seu contra lateral.

Existem muitos fatores que influenciam no sucesso da reparação da fratura em que é usada a cerclagem. Estes incluem a configuração das congruências das fraturas, número de cerclagens aplicadas e a habilidade da cerclagem em resistir às forças aplicadas (ROE, 1997). Neste trabalho, apesar da fratura ser transversal e sem congruências, as duas cerclagens (uma proximal e uma distal à fratura) foram suficientes para proporcionar estabilidade no local da mesma e resistir às forças aplicadas, haja vista não ocorrer a ruptura de pinos e/ou fios metálicos durante os testes.

A partir dos resultados obtidos, a utilização do método de transfixação interna deve ser estudada experimentalmente in vivo, antes de ser indicada para a rotina cirúrgica veterinária.

\section{CONCLUSÃO}

A análise mediante a utilização da prensa Soloteste ${ }^{\circledR}$ para a avaliação dos efeitos das forças de compressão axial e flexão com o uso de pino intramedular ou transfixação esquelética interna permite admitir que a técnica de transfixação esquelética interna proporciona maior rigidez que a do pino intramedular em relação às forças de compressão axial e envergamento; o tempo médio de 21,4min para a elaboração do dispositivo de transfixação esquelética interna, superior ao de pinos intramedulares $(5,36 \mathrm{~min})$, não inviabiliza a sua utilização na cirurgia.

\section{AGRADECIMENTOS}

Agradecemos ao Engenheiro Paulo Inácio Obregon do Carmo e ao funcionário João Francisco Nunes Maciel pelas orientações prestadas quanto à utilização da prensa durante os testes.

\section{FONTES DE AQUISIÇÃO}

a - Prensa Soloteste ${ }^{\circledR}$, para testes de resistência de materiais, São Paulo - SP.

b - Jet acrílico auto polimerizante - Clássico artigos odontológicos Ltda. Av. Diógines Ribeiro de Lima , 2720. São Paulo - SP

c - Abraçadeiras de náilon Vonder. O.V.D. Importadora e distribuidora Ltda. WWW.vonder.com.br

\section{REFERÊNCIAS BIBLIOGRÁFICAS}

BERNARDE, A. et al. An in vitro biomechanical study of bone plate and interlocking nail in a canine diaphyseal femoral fracture model. Veterinary Surgery, v.30, n.5, p.397408, 2001

DAMIÁN Z. et al. Device for biomechanical torsion testes of long bones in an instron test machine. Capturado em 16 mai. 2003. Online. Disponível na Internet: http://www.cistrun.unam.mx/ revista/pdfv3n7/artic5.pdf

FOSSUM, T.W. Fundamentos da cirurgia ortopédica e tratamento de fraturas. In:_-_-_. Cirurgia de pequenos animais. São Paulo: Roca, 2002. Cap.28, p. 794 .

GILMORE, D.R. Fixação interna de fraturas femurais. In: BOJRAB, M.J. Técnicas atuais em cirurgia de pequenos animais. 3.ed. São Paulo : Roca, 1996. Cap.46, p.642-651.

HULSE, D.; HYMAN, B. Biologia e biomecânica das fraturas. In: SLATTER, D. Manual de cirurgia de pequenos animais. São Paulo : Manole, 1998. V.2, cap.120, p.1893-1984.

MARKEL, M.D. et al. Mechanical properties of long bones in dogs. American Journal Veterinary Research, v.55, n.8, p.1178-1183, 1994.

PIERMATTEI, D.L.; FLO, G.L. Manual de ortopedia e tratamento das fraturas dos pequenos animais. 3.ed. São Paulo : Manole, 1999. Cap.16, p.437-479.

ROE, S.C. Mechanical characteristics and comparisons of cerclage wires: Introduction of the double-wrap and loop/ twist tying methods. Veterinary Surgery, v.26, n.4, p.310316, 1997.

SCHMIDT, S.S. et al. Biomechanical evaluation of fracture fixation methods. 1994. Capturado em 18 maio. 2003. Online. Disponível na Internet: http://www.Guide.stanford.edu/pubblication/dev7.html $-7 \mathrm{k}$

SUKHIANI, H.R. et al. Ex vivo biomechanical comparison of pin fixation techniques for canine distal femoral physeal fractures. Veterinary Surgery, v.26, n.5, p.398-407, 1997.

Ciência Rural, v.35, n.1, jan-fev, 2005. 